ANNALES

POLONICI MATHEMATICI

$81.1(2003)$

\title{
Existence of positive solutions for a nonlinear fourth order boundary value problem
}

\author{
by Ruyun MA (Gansu)
}

$$
\begin{aligned}
& \text { problem } \\
& \qquad \begin{array}{l}
u^{(4)}(x)=\lambda a(x) f(u(x)), \\
u(0)=u^{\prime}(0)=u^{\prime \prime}(1)=u^{\prime \prime \prime}(1)=0,
\end{array}
\end{aligned}
$$

Abstract. We study the existence of positive solutions of the nonlinear fourth order

where $a:[0,1] \rightarrow \mathbb{R}$ may change sign, $f(0)>0$, and $\lambda>0$ is sufficiently small. Our approach is based on the Leray-Schauder fixed point theorem.

1. Introduction. The deformations of an elastic beam are described by a fourth order two-point boundary value problem [5]. The boundary conditions are given according to the controls at the ends of the beam. For example, the nonlinear fourth order problem

$$
\begin{aligned}
& u^{(4)}(x)=\lambda a(x) f(u(x)), \\
& u(0)=u^{\prime}(0)=u^{\prime \prime}(1)=u^{\prime \prime \prime}(1)=0,
\end{aligned}
$$

describes the deformations of an elastic beam with one end fixed and the other end free.

The existence of solutions of $(1.1)_{\lambda}$ has been studied by Gupta [5]. But to the best of our knowledge, there are no results concerning the existence of positive solutions of $(1.1)_{\lambda}$. In this paper, we will study this problem.

We make the following assumptions:

(A1) $f: \mathbb{R}^{+} \rightarrow \mathbb{R}$ is continuous and $f(0)>0$.

(A2) $a$ is continuous on $[0,1], a \equiv 0$ does not hold on $[0,1]$ and there

2000 Mathematics Subject Classification: Primary 34B18.

Key words and phrases: three-point boundary value problem, positive solution, LeraySchauder fixed point theorem.

Supported by the Foundation for Major Projects in Science and Technology of the Education Ministry of China, GG-110-10736-1003, NWNU-KJCXGC-212 and the NSFC (10271095). 
exists a number $k>1$ such that

$$
\int_{0}^{x}\left(\int_{0}^{\tau}\left(\int_{r}^{1}\left[\int_{s}^{1}\left(a^{+}(t)-k a^{-}(t)\right) d t\right] d s\right) d r\right) d \tau \geq 0, \quad x \in[0,1],
$$

where $a^{+}$is the positive part of $a$ and $a^{-}$is the negative part of $a$.

Our main result is

TheOREM 1. Let (A1) and (A2) hold. Then there exists a positive number $\lambda^{*}$ such that $(1.1)_{\lambda}$ has at least one positive solution for $0<\lambda<\lambda^{*}$.

REMARK. We note that condition (A2) is meaningful. Take

$$
b(x)= \begin{cases}9 / 10-x, & x \in[0,9 / 10] \\ \frac{9-10 x}{10 k}, & x \in[9 / 10,1]\end{cases}
$$

where $k \in(1, \infty)$ is a constant. It is easy to check that $b^{+}(x)-k b^{-}(x)=$ $9 / 10-x$ for $x \in[0,1]$. Moreover

$$
\begin{aligned}
\int_{0}^{x}\left(\int _ { 0 } ^ { \tau } \left(\int_{r}^{1}\left[\int_{s}^{1}\left(b^{+}(t)-k b^{-}(t)\right) d t\right]\right.\right. & d s) d r) d \tau \\
& =\int_{0}^{x}\left(\int_{0}^{\tau}\left(\int_{r}^{1}\left[\int_{s}^{1}(9 / 10-t) d t\right] d s\right) d r\right) d \tau \\
& =\frac{7 x^{2}}{120}-\frac{x^{3}}{15}+\frac{3 x^{4}}{80}+\frac{x^{5}}{120} \geq 0
\end{aligned}
$$

for $x \in[0,1]$ and $k \in(1, \infty)$. This means that $b$ satisfies (A2).

The proof of our Theorem 1 is based upon the Leray-Schauder fixed point theorem and motivated by [6]. In [6], Hai studied the existence of positive solutions for the elliptic equation

$$
\begin{aligned}
& \Delta u+\lambda a(t) g(u)=0, \\
& \left.u\right|_{\partial \Omega}=0,
\end{aligned}
$$

where a may change sign. In [1], Castaneda and Ma proved an analogous result for a second order three-point boundary value problem. For other results on the existence and multiplicity of positive solutions of fourth order ordinary differential equations with other boundary conditions, one may refer, with further references therein, to Dunninger [3], Graef and Yong [4], Ma and Wang [7], Del Pino and Manásevich [2] and Zhang and Kong [8].

2. Preliminary lemmas. To prove Theorem 1, we need the following preliminary results. 
Lemma 1. For $y \in C[0,1]$, the problem

$$
\begin{aligned}
& u^{(4)}(x)=y(x), \quad x \in(0,1), \\
& u(0)=u^{\prime}(0)=u^{\prime \prime}(1)=u^{\prime \prime \prime}(1)=0,
\end{aligned}
$$

is equivalent to the integral equation

$$
u(x)=\int_{0}^{x}\left(\int_{0}^{\tau}\left(\int_{r}^{1}\left[\int_{s}^{1} y(t) d t\right] d s\right) d r\right) d \tau .
$$

Moreover, if $y \geq 0$ on $[0,1]$, then

$$
u \geq 0, \quad t \in[0,1] .
$$

Proof. It is easy to check that (2.1) is equivalent to (2.2). (2.3) is an immediate consequence of $(2.2)$ and the condition that $y \geq 0$ on $[0,1]$.

Lemma 2. Let (A1) and (A2) hold. Then for every $0<\delta<1$, there exists a positive number $\bar{\lambda}$ such that, for $0<\lambda<\bar{\lambda}$, the problem

$$
\begin{aligned}
& u^{(4)}(x)=\lambda a^{+}(x) f(u(x)), \quad x \in(0,1), \\
& u(0)=u^{\prime}(0)=u^{\prime \prime}(1)=u^{\prime \prime \prime}(1)=0,
\end{aligned}
$$

has a positive solution $\widetilde{u}_{\lambda}$ with $\left|\widetilde{u}_{\lambda}\right|_{0} \rightarrow 0$ as $\lambda \rightarrow 0$, and

$$
\widetilde{u}_{\lambda}(x) \geq \lambda \delta f(0) p(x), \quad x \in[0,1],
$$

where

$$
p(x)=\int_{0}^{x}\left(\int_{0}^{\tau}\left(\int_{r}^{1}\left[\int_{s}^{1} a^{+}(t) d t\right] d s\right) d r\right) d \tau .
$$

Proof. We know that $p(x) \geq 0$ for $x \in[0,1]$. By Lemma 1, (2.4) is equivalent to the integral equation

$$
u(x)=\lambda \int_{0}^{x}\left(\int_{0}^{\tau}\left(\int_{r}^{1}\left[\int_{s}^{1} a^{+}(t) f(u(t)) d t\right] d s\right) d r\right) d \tau=: A u(x)
$$

where $u \in C[0,1]$. Then $A: C[0,1] \rightarrow C[0,1]$ is completely continuous, and fixed points of $A$ are solutions of (2.4). We shall apply the Leray-Schauder fixed point theorem to prove that $A$ has a fixed point for $\lambda$ small.

Let $\varepsilon>0$ be such that

$$
f(t) \geq \delta f(0) \quad \text { for } 0 \leq t \leq \varepsilon .
$$

Suppose that

$$
\lambda<\frac{\varepsilon}{2|p|_{0} \widetilde{f}(\varepsilon)}=: \bar{\lambda}
$$


where $\widetilde{f}(t)=\max _{0 \leq s \leq t} f(s)$. Since $\lim _{t \rightarrow 0^{+}} \widetilde{f}(t) / t=\infty$ it follows that there exists $r_{\lambda} \in(0, \varepsilon)$ such that

$$
\frac{\widetilde{f}\left(r_{\lambda}\right)}{r_{\lambda}}=\frac{1}{2 \lambda|p|_{0}} .
$$

We note that this implies $r_{\lambda} \rightarrow 0$ as $\lambda \rightarrow 0$.

Now, we consider the homotopy equations

$$
u=\theta A u, \quad \theta \in(0,1) .
$$

Let $u \in C[0,1]$ and $\theta \in(0,1)$ be such that $u=\theta A u$. We claim that $|u|_{0} \neq r_{\lambda}$. In fact

$$
u(x)=\theta \lambda \int_{0}^{x}\left(\int_{0}^{\tau}\left(\int_{r}^{1}\left[\int_{s}^{1} a^{+}(t) f(u(t)) d t\right] d s\right) d r\right) d \tau
$$

so we have $|u|_{0} \leq \lambda|p|_{0} \widetilde{f}\left(|u|_{0}\right)$, or

$$
\frac{\widetilde{f}\left(|u|_{0}\right)}{|u|_{0}} \geq \frac{1}{\lambda|p|_{0}}
$$

which implies that $|u|_{0} \neq r_{\lambda}$. Thus by the Leray-Schauder fixed point theorem, $A$ has a fixed point $\widetilde{u}_{\lambda}$ with

$$
\left|\widetilde{u}_{\lambda}\right|_{0} \leq r_{\lambda}<\varepsilon .
$$

Moreover, combining (2.6)-(2.8) and Lemma 1, we obtain (2.5).

3. Proof of Theorem 1. Let

$$
q(x)=\int_{0}^{x}\left(\int_{0}^{\tau}\left(\int_{r}^{1}\left[\int_{s}^{1} a^{-}(t) f(u(t)) d t\right] d s\right) d r\right) d \tau .
$$

Then $q(x) \geq 0$ on $[0,1]$. By (A2), there exist $c, d \in(0,1)$ such that

$$
q(x)|f(y)| \leq d p(x) f(0)
$$

for $y \in[0, c]$ and $x \in[0,1]$. Fix $\delta \in(d, 1)$ and let $\lambda^{*}>0$ be such that

$$
\left|\widetilde{u}_{\lambda}\right|_{0}+\lambda \delta f(0)|p|_{0} \leq c
$$

for $\lambda<\lambda^{*}$, where $\widetilde{u}_{\lambda}$ is given by Lemma 2 , and

$$
|f(\alpha)-f(\beta)| \leq f(0) \frac{\delta-d}{2}
$$

for $\alpha, \beta \in[-c, c]$ with $|\alpha-\beta| \leq \lambda^{*} \delta f(0)|p|_{0}$. 
Let $\lambda<\lambda^{*}$. We look for a solution $u_{\lambda}$ of the form $\widetilde{u}_{\lambda}+v_{\lambda}$. Here $v_{\lambda}$ solves

$$
\begin{gathered}
v^{(4)}(x)=\lambda a^{+}(x)\left(f\left(\widetilde{u}_{\lambda}+v\right)-f\left(\widetilde{u}_{\lambda}\right)\right) \\
-\lambda a^{-}(x) f\left(\widetilde{u}_{\lambda}+v\right), \quad x \in(0,1), \\
v(0)=v^{\prime}(0)=v^{\prime \prime}(1)=v^{\prime \prime \prime}(1)=0 .
\end{gathered}
$$

For each $w \in C[0,1]$, let $v=T(w)$ be the solution of

$$
\begin{aligned}
& v^{(4)}(x)=\lambda a^{+}(x)\left(f\left(\widetilde{u}_{\lambda}+w\right)-f\left(\widetilde{u}_{\lambda}\right)\right)-\lambda a^{-}(x) f\left(\widetilde{u}_{\lambda}+w\right), \quad x \in(0,1), \\
& v(0)=v^{\prime}(0)=v^{\prime \prime}(1)=v^{\prime \prime \prime}(1)=0 .
\end{aligned}
$$

Then $T: C[0,1] \rightarrow C[0,1]$ is completely continuous.

Let $v \in C[0,1]$ and $\theta \in(0,1)$ be such that $v=\theta T v$. Then we have

$$
\begin{aligned}
& v^{(4)}(x)=\theta \lambda a^{+}(x)\left(f\left(\widetilde{u}_{\lambda}+v\right)-f\left(\widetilde{u}_{\lambda}\right)\right)-\theta \lambda a^{-}(x) f\left(\widetilde{u}_{\lambda}+v\right), \quad x \in(0,1), \\
& v(0)=v^{\prime}(0)=v^{\prime \prime}(1)=v^{\prime \prime \prime}(1)=0 .
\end{aligned}
$$

We claim that $|v|_{0} \neq \lambda \delta f(0)|p|_{0}$. Suppose to the contrary that $|v|_{0}=$ $\lambda \delta f(0)|p|_{0}$. Then by (3.3) and (3.4), we obtain

$$
\left|\widetilde{u}_{\lambda}+v\right|_{0} \leq\left|\widetilde{u}_{\lambda}\right|_{0}+|v|_{0} \leq c
$$

and

$$
\left|f\left(\widetilde{u}_{\lambda}+v\right)-f\left(\widetilde{u}_{\lambda}\right)\right|_{0} \leq f(0)\left(\frac{\delta-d}{2}\right) .
$$

Using (3.2) and (3.5)-(3.7) and applying Lemma 1, we have

$$
\begin{aligned}
|v(x)| & \leq \lambda \frac{\delta-d}{2} f(0) p(x)+\lambda f\left(\left|\widetilde{u}_{\lambda}+v\right|_{0}\right) q(x) \\
& \leq \lambda \frac{\delta-d}{2} f(0) p(x)+\lambda d f(0) p(x) \\
& =\lambda \frac{\delta+d}{2} f(0) p(x), \quad x \in(0,1) .
\end{aligned}
$$

In particular

$$
|v|_{0} \leq \lambda \frac{\delta+d}{2} f(0)|p|_{0}<\lambda \delta f(0)|p|_{0},
$$

a contradiction, and the claim is proved. Thus by the Leray-Schauder fixed point theorem, $T$ has a fixed point $v_{\lambda}$ with $\left|v_{\lambda}\right|_{0} \leq \lambda \delta f(0)|p|_{0}$. Using (2.5) and (3.8), we obtain

$$
\begin{aligned}
u_{\lambda}(x) & \geq \widetilde{u}_{\lambda}(x)-\left|v_{\lambda}(x)\right| \geq \lambda \delta f(0) p(x)-\lambda \frac{\delta+d}{2} f(0) p(x) \\
& =\lambda \frac{\delta-d}{2} f(0) p(x) .
\end{aligned}
$$

Thus, $u_{\lambda}$ is a positive solution of $(1.1)_{\lambda}$. 


\section{References}

[1] N. Castaneda and R. Y. Ma, Positive solutions to a second order three-point boundary value problem, Appl. Anal. 76 (2000), 231-239.

[2] M. R. Del Pino and R. F. Manásevich, Existence for a fourth-order boundary value problem under a two-parameter nonresonance condition, Proc. Amer. Math. Soc. 112 (1991), 81-86.

[3] D. R. Dunninger, Multiplicity of positive solutions for a nonlinear fourth order equation, Ann. Polon. Math. 77 (2001), 161-168.

[4] J. R. Graef and B. Yong, Positive solutions of a boundary value problem for fourth order nonlinear differential equations, Proc. Dynamic Systems Appl. 3 (2000), 217224 .

[5] C. P. Gupta, Existence and uniqueness theorems for the bending of an elastic beam equation, Appl. Anal. 26 (1988), 289-304.

[6] D. D. Hai, Positive solutions to a class of elliptic boundary value problems, J. Math. Anal. Appl. 227 (1998), 195-199.

[7] R. Y. Ma and H. Y. Wang, On the existence of positive solutions of fourth-order ordinary differential equations, Appl. Anal. 59 (1995), 225-231.

[8] B. G. Zhang and L. J. Kong, Positive solutions of fourth-order singular boundary value problems, Nonlinear Stud. 7 (2000), 70-77.

Department of Mathematics

Northwest Normal University

Lanzhou 730070, Gansu, P. R. China

E-mail: mary@nwnu.edu.cn 